\title{
Detailed Comparison between Parton Cascade and Hadronic Cascade at SPS and RHIC *
}

\author{
Yasushi Nara ${ }^{1,2}$, Klaus Geiger ${ }^{1}$ and Ronald Longacre ${ }^{1}$ \\ ${ }^{1}$ Physics Department, Brookhaven National Laboratory, Upton, N.Y. 11979, U.S.A. \\ 2 Advanced Science Research Center, Japan Atomic Energy Research Institute, \\ Tokai, Naka, Ibaraki 319-1195, Japan
}

\begin{abstract}
We study the importance of the partonic phase produced in relativistic heavy ion collision by comparing the parton cascade model and the hadronic cascade model. Hadron yield, baryon stopping and transverse momentum distribution are calculated with JAM and discussions are given comparing with VNI. Both of these models give good description of experimental data. We also discuss the strangeness production mechanism and the directed transverse flow.
\end{abstract}

\section{INTRODUCTION}

Hadronic transport models (also called hadronic cascade) have been used to investigate the many properties of hot and dense matter produced in heavy ion collisions. The models are extremely successful and can explain many observables. Most hadronic cascade models such as RQMD [1], QGSM [2], UrQMD [3] and HSD [4] are based on string phenomenology which simulates soft interactions among hadrons. Partonic degrees of freedom are treated implicitly, although deconfinement is not included. For example, hadrons which have constituent quarks within a formation time can collide with additive quark cross sections. In this manner, the effect of quark-quark interactions are also included in hadronic transport models. A detailed analysis of the partonic contribution in UrQMD can be found in Ref. [5].

Event generators based on perturbative QCD ( $p Q C D$ ) have been proposed such as HIJING [6] and VNI [7], in order to describe ultra-relativistic heavy ion collisions, especially, at collider energies ( $\mathrm{RHIC} / \mathrm{LHC}$ ). VNI is a Monte Carlo implementation of parton cascade model (PCM) in which the time evolution of heavy ion collision is simulated by the parton cascading. Recently VNI has been applied to nuclear reactions at SPS energies [8] and it is found that parton picture well describes experimental data at SPS energies.

* This work was supported in part by U.S. Department of Energy Contract No. DE-AC02-98CH10886. 
The purpose of this work is to study the partonic contribution using VNI which is a transport model based on parton picture and hadrons from a string theory based model JAM [9].

\section{PARTON CASCADE V.S. HADRONIC CASCADE}

It is known that both the parton cascade model and the hadronic cascade model well describe the global structure of the heavy ion collisions at SPS. i.e. transverse energy production, rapidity and transverse distribution. Here we will explain briefly both the parton cascade model VNI and hadronic cascade model JAM. The Monte Carlo parton cascade model VNI [7] has been developed by Klaus Geiger for the purpose to explore the the space-time evolution of partons in high energy collisions using pQCD. This model may be suitable to describe ultra-relativistic heavy ion collisions at collider energies, such as BNL-RHIC and CERN-LHC, and it has met some successes in describing heavy ion collisions even at CERN-SPS energies [8]. The detailed description can be found in Ref. [7]. Actual simulation procedure of VNI is (1) Sampling partons according the experimentally measured nucleon structure functions. (2) Parton cascading based on pQCD including initial and final state radiations. (3) Hadronization according to parton-cluster formation. (4) Decay of parton cluster and beam cluster.

The main features included in JAM are as follows. (1) At low energies, inelastic hadron-hadron collisions are modeled by the resonance productions based on the idea from RQMD and UrQMD. (2) Above the resonance region, soft string excitation is implemented along the lines of the HIJING model [6]. (3) Multiple minijet production is also included in the same way as the HIJING model in which jet cross section and the number of jet is calculated using an eikonal formalism for pQCD and hard parton-parton scatterings with initial and final state radiation are simulated using PYTHIA [10] program. Same parameters as HIJING are used in terms of soft and hard processes. (4) Rescattering of hadrons which have original constituent quarks can scatter again with other hadrons assuming the additive quark cross section within a formation time. (5) At around SPS energies, within our parameterization dominant interactions are soft string excitation. (6) rescattering among on-shell partons from hard scattering are not included.

Figure 1 shows the example of the hadronic cascade results of the net proton, charged particle and the transverse energy rapidity distribution from JAM in $\mathrm{Pb}+\mathrm{Pb}$ collision at SPS energy. The experimental baryon stopping, the yield of produced particles and transverse energy production are well accounted for in JAM in nucleus-nucleus collisions a1 SPS energy. JAM predicts no baryon free region at midrapidity. A good description has also been obtained with VNI [8]. JAM results are similar to RQMD and UrQMD calculations $[15,3]$. Both constituent quark scatterings $[15,3]$ and diquark breaking $[17,18]$ are important for the explanation of baryon stopping at SPS energies in hadronic cascade picture.

In order to reproduce experimental data of transverse energy production, rescat- 
rescattering. The rescattering effect is not visible in both net protons and produced negative charged particles in target rapidity region. Inclusion of rescattering among hadrons improve only the results at mid-rapidity region and the agreement is very good for both net protons and negative charged particles.

\section{STRANGENESS PRODUCTION}

Strangeness enhancement in heavy ion collisions compared to $p p$ collisions has been predicted as a signal of a QGP [16]. At the AGS energies, it has been found that rescattering can explain the strangeness enhancement (RQMD and ARC), while at SPS energies a different mechanism is necessary.

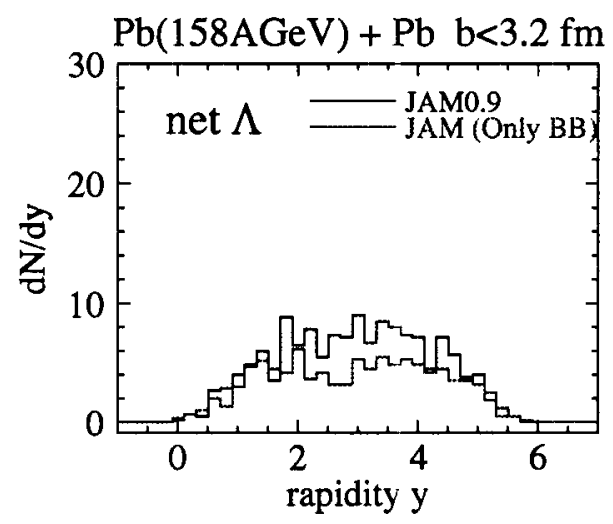

FIGURE 3. Rapidity distribution of net $\Lambda$ calculated with JAM for $\mathrm{Pb}+\mathrm{Pb}$ collision at $158 \mathrm{GeV} / \mathrm{c}$.

The sources of the strange quarks are $s \bar{s}$ creations from string fragmentation and rescattering among hadrons in JAM. While in the parton cascade model, strange quarks are produced from hard parton-parton collisions (in this work, we do not include soft parton-parton collision contributions and final state interaction among hadrons) and parton clusters as well as beam clusters.

The simple string based morlels produce less strangeness than the experimental data at SPS energies. If we include final state interactions among hadrons plus collective effects such as string fusion $[12,18]$, one can explain the experimental yield.

In this section, we study tirne evolution of strange quark using VNI. In Fig 4, we show in the right panel the time evolution of the ratio of $s \bar{s}$ pairs to average light quark pairs during the parton cascade in the VNI Monte Carlo model at SPS energies $\left(R_{s}=\frac{2 N(s \bar{s})}{N(u \bar{u})+N(d d)}\right)$. VNI predicts that the ratio of created strange quark pairs is around $10 \%$ in partonic phase. This value is similar to the prediction by RQMD without rescattering [1]. 

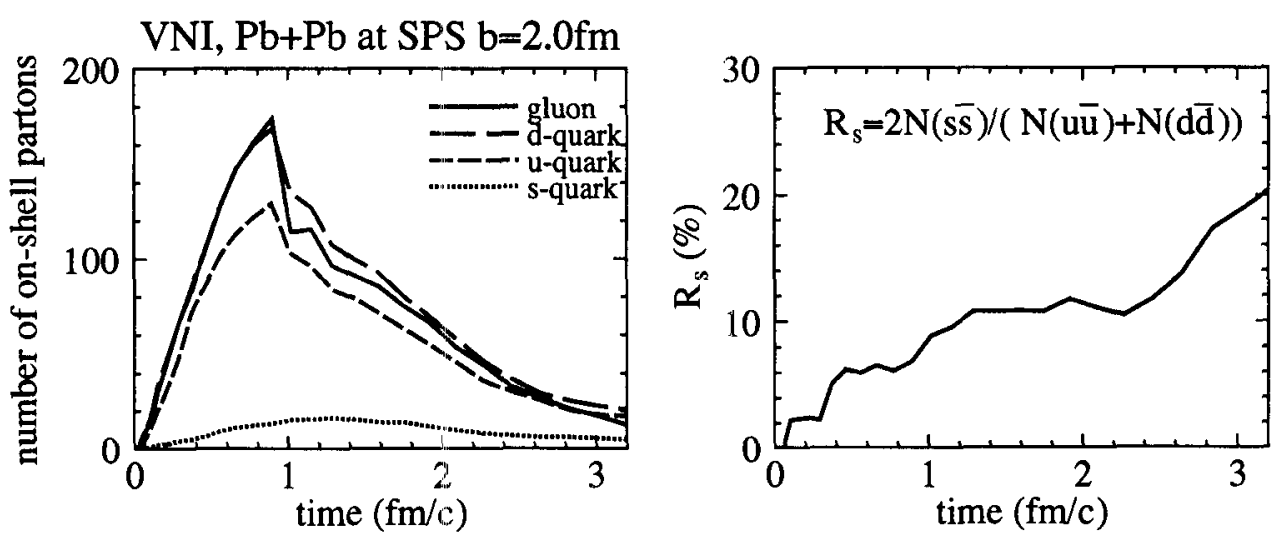

FIGURE 4. Time evolutions of produced partons from VNI for $\mathrm{Pb}+\mathrm{Pb}$ collision at $158 \mathrm{GeV} / \mathrm{c}$. Solid line, long-dashed line, short-dashed and dotted line correspond to gluons, d-quarks, u-quarks and s-quarks respectively.

In fig 5 , we show the time evolutions of produced partons at RHIC energy. It is seen that the yield of strange quark is much larger in comparison with SPS energy. However chemical equilibration of parton system is not expected within the parton cascade model.

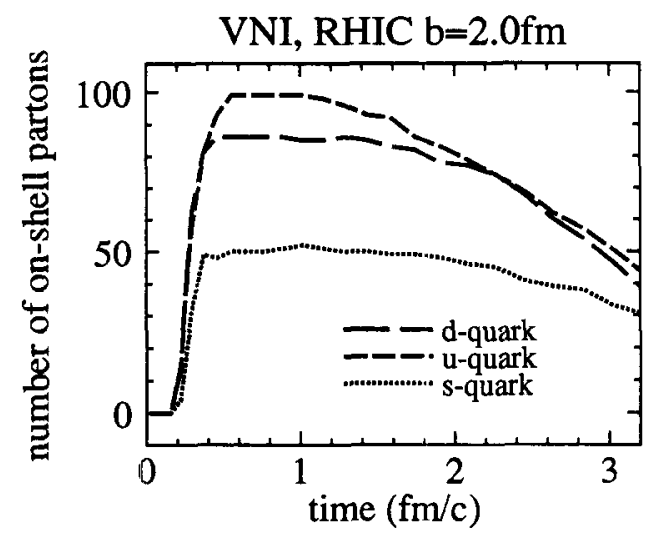

FIGURE 5. Time evolutions of produced partons from VNI for $\mathrm{Au}+\mathrm{Au}$ collision at RHIC. 


\section{DIRECTED TRANSVERSE FLOW AT SPS}

It has been argued that flow is sensitive to the equation of state (EoS) of strongly interacting matter. Recently a directed transverse flow at SPS energy has been found by the WA98 [14] collaboration using the Plastic Ball detector. They have pointed out that the magnitucle of the directed flow is two or three times smaller than RQMD [1] and VENUS [17] model predictions. Hydrodynamical model calculations indicate that EoS which simulates QGP phase transition reduces the directed transverse flow [19] in compared with hadronic EoS case.

JAM prediction as shown is Fig.6 gives similar results to RQMD calculation. The directed transverse flow which is defined by

$$
p_{x}^{d i r}=\int\left|\frac{d p_{x}}{d y}\right| d y
$$

is plotted in Fig 7 as a function of time in JAM. The calculation which includes only baryon-baryon collisions shows no transverse flow in JAM. It is seen that within a hadronic transport model, the origin of the directed flow is hadronic rescattering.

On the other hand, we have lound that parton cascade model gives partonic flow generated by parton-parton cascading. Detailed investigation with VNI including hadronic rescattering is in progress. We expect that the time evolution of flow in the parton plus hadronic rescattering phase will be different than that of the pure hadron cascade code.

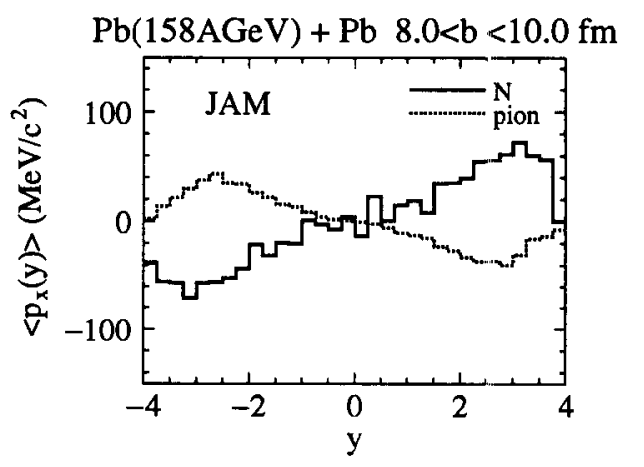

FIGURE 6. The transverse flow of nucleons (solid line) and pions (dotted line) for $\mathrm{Pb}+\mathrm{Pb}$ collision at $158 \mathrm{GeV} / \mathrm{c}$ with impact parameter $8 \mathrm{fm}<b<10 \mathrm{fm}$.

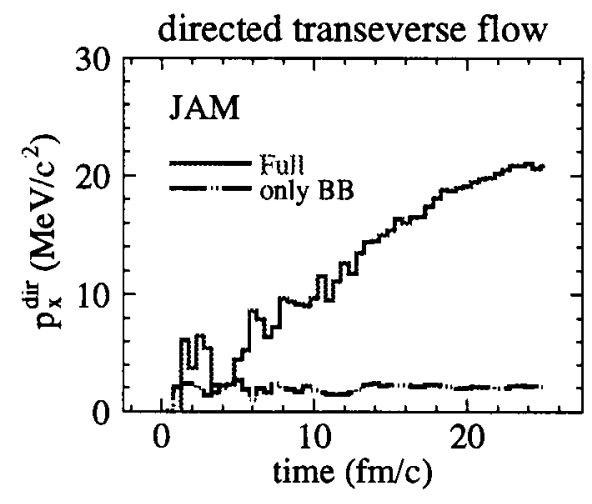

FIGURE 7. Time evolution of the directed transverse flow in JAM (full line). Dash-dotted line corresponds to the result without hadronic rescattering. 


\section{SUMMARY}

We have discussed the partonic contribution by comparing the predictions of both parton cascade model VNI based on PQCD parton picture and hadronic cascade model JAM based on the string picture.

Both hadronic and partonic cascade model well describe particle spectra at SPS energies, however, hadronic cascade model JAM overpredicts the strength of the directed transverse flow. It has been shown that in Ref. $[20,21]$ within a hadronic transport model, flow depend; strongly on the mean filed which simulate phase transition at AGS energies. It is interesting to investigate the mean filed effects at SPS using BUU model.

Concerning strangeness production, parton cascade model gives the similar $R_{s}$ value to that of RQMD2.3 [1] with rope formation (without rescattering). The result indicates that parton picture seems to explain strangeness abundance. In the next work, we will study the mechanism of baryon stopping and the transverse flow within the parton cascade model.

\section{ACKNOWLEDGMENTS}

One of us (Y.N) would like to acknowledge the hospitality of Brookhaven National Laboratory and there I had started to work with Klaus. He always stimulated and encouraged me and I learned much about his philosophy of physics from him. I will never forget him.

\section{REFERENCES}

1. H. Sorge, Phys. Rev. C 52, 3291 (1995).

2. L. Bravina, L.P. Csernai, P. Levai, and D. Dtrottman, Phys. Rev. C 51, 2161 (1994).

3. L.A. Winckelmann, S.A. Bass, M. Bleicher, M. Brandstetter, A. Dumitru, C. Ernst, L. Gerland, J. Konopka, S. Soff, C. Spieles, H. Weber, C. Hartnack, J. Aichelin, N. Amelin, H. Stöcker and W. Greiner, Nucl. Phys. A610, 116c (1996); nuclth/9610033; S.A. Bass, M. Belkacem, M. Bleicher, M. Brandstetter, L. Bravina, C. Ernst, L. Gerland, M. Hofmann, S. Hofmann, J. Konopka, G. Mao, L. Neise, S. Soff, C. Spieles, H. Weber, L.A. Winckelmann, H. Stöcker, W. Greiner, C. Hartnack, J. Aichelin and N. Amelin, I'rog. Part. Nucl. Phys. 41, 225 (1998); nucl-th/9803035.

4. W. Ehehalt and W. Cassing Nucl. Phys. A602, 449 (1996); J. Geiss, W. Cassing and C. Greiner, nucl-th/9805012.

5. H. Weber, C. Ernst, M. Bleicher, L. Bravina, H. Stöcker and W. Greiner, C. Spieles and S. A. Bass, nucl-th/9808021.

6. X. N. Wang, Phys. Rep. 280, 287 (1997); X. N. Wang and M. Gyulassy, Comp. Phys. Comm. 83, 307 (1994); http://www-nsdth.lbl.gov/ xnwang/hijing/.

7. K. Geiger, Phys. Rep. 258, 238 (1995); Comp. Phys. Comm. 104, 70 (1997); http://penguin.phy.bnl.gov/־klaus/, nucl-th/9806102. 
8. K. Geiger and D. K. Srivastava, Phys. Rep. C56, 2718 (1997);

D. K. Srivastava and K. Geiger, Phys. Lett. B422, 39 (1998);

K. Geiger and R. Longacre, Heavy Ion Physics 8, 41 (1998).

9. Y. Nara, Nucl. Phys. A638, 555c (1998); JAM1.0 manual to be submitted to Comp.Phys. Comm.

10. T. Sjöstrand, Comp. Phys. Comm. 82, 74 (1994); http://www.thep.lu.se/tf2/staff/torbjorn/Pythia.html.

11. B. Andersson, G. Gustafson and H. Pi, Z. Phys. C 57, 485 (1993);

H. Pi, Comp. Phys. Comm. 71, 173 (1992).

12. H. Sorge, L. A. Winckelmann, H. Stöcker and W. Greiner, Z. Phys. C 59, 85 (1993).

13. J. Bächler et al., Phys. Rev. Lett. 72, 1419 (1994).

14. S. Nishimura et al., Nucl. Phys. A638, 549 (1998).

15. H. Sorge, A. V. Keitz, R. Mattiello, H. Stöker and W. Greiner, Z. Phys. C 47, 629 (1990).

16. P. Koch, B. Müller and J. Rafelski, Phys. Rep. 142, 167 (1986).

17. K. Werner, Phys. Rep. 232, 87 (1993).

18. S.E. Vance, M. Gyulassy and X.N. Wang, Phys. Lett. B443, 45 (1998).

19. N. S. Amelin, et al., Phys. Rev. Lett. 67, 1523 (1991).

20. P. Danielewicz, Roy A. Lacey, P.B. Gossiaux, C. Pinkenburg, P. Chung, J.M. Alexander, R.L. McGrath. Mar Phys. Rev. Lett. 81, 2438 (1998).

21. Bao-An Li and C. M. Ko, Phys. Rev. C 58, R1382 (1998). 